\title{
Late stent thrombosis in drug-eluting stents: return of the 'VB syndrome'
}

\author{
Patrick W Serruys and Neville Kukreja
}

In the 1990s, when vascular brachytherapy (VB) was proposed as the definitive 'cure' for restenosis, we embraced the technology with great enthusiasm, but quickly discovered that there was a dark side. Our study group was the first to report an abnormal incidence of late thrombosis in the post-VB population, triggering a wave of reports. The late complication rate, the complexity of the procedure's logistics and the advent of the drug-eluting stent (DES) killed off VB in its infancy. Even the pioneers of the technique recognized that we were sitting on a time bomb. At the European Society of Cardiology (ESC) annual meeting in Barcelona this year, we experienced a revival of the 'VB syndrome', only this time DESs were at the center of the dispute. The ESC newsletter ran a headline of "Do drug-eluting stents increase deaths?" So, what are the facts?

Two years ago in The Lancet we reported four cases of late thrombosis following DES implantation after antiplatelet therapy was discontinued-following recommendations by doctors. Concerned by this experience, two large institutions (University Hospital, Bern, Switzerland and the Thoraxcenter, Erasmus Medical Center, Rotterdam, the Netherlands) combined their experience and reviewed data from 8,146 patients to establish the incidence of early ( $\leq 30$ days) and late ( $>30$ days) angiographic stent thrombosis. These data became public at the ESC meeting. The cumulative incidence of stent thrombosis was $2.9 \%$ after 3 years. Late stent thrombosis occurred at a constant rate, with an incidence of $0.6 \%$ per year between 30 days and 3 years with no evidence of diminution. This phenomenon was observed with both sirolimus-eluting and paclitaxel-eluting stents. The univariate predictors of stent thrombosis were acute coronary syndrome at presentation, bifurcation treatment, number of stents and total stent length. In hospital, $7 \%$ of the patients with stent thrombosis died; $9 \%$ of these $(n=8)$ ....in Barcelona

this year, we

experienced a

revival of the

'VB syndrome', only this time

DESs were at

the center

of the dispute.

PW Serruys is Chief of the Interventional Cardiology Section and N Kukreja is an interventional cardiology fellow at the Thoraxcenter, Erasmus Medical Center, Rotterdam, the Netherlands. $P W$ Serruys is also an Advisory Board member of Nature Clinical Practice

Cardiovascular Medicine.

\section{Competing interests}

The authors declared they have no competing interests.

www.nature.com/clinicalpractice doi:10.1038/ncpcardio0722 because of early and $5 \%(n=3)$ because of late thrombosis. In other words, of more than 8,000 patients, only 3 died from late thrombosis. We must realize, however, that angiographically documented stent thrombosis could represent just the tip of the iceberg.

A meta-analysis of published, industrysponsored, randomized trials that examined late stent thrombosis 1-12 months after implantation, concluded that the rate of late stent thrombosis in patients with DESs was no different from that in patients who received bare-metal stents $(0.23 \%$ and $0.25 \%$, respectively). In a large industry-sponsored postmarketing surveillance study of 13,437 patients receiving DESs, late stent thrombosis within 1 year was $0.2 \%$; however, in another 12,049 patients also receiving DESs (reported by six different institutions) the incidence was in the range $0.3-0.9 \%$, rising to $1.8 \%$ in patients with multivessel disease. To put these numbers into context it is appropriate to quote the incidence of late stent thrombosis in bare-metal stents, which is in the range $0.4-0.8 \%$ in a total of 9,465 patients reported in the literature.

Sudden death and recognized/unrecognized myocardial infarction as a potential surrogate for late stent thrombosis needs further investigation. Recently, European and North American cardiologists, members of the European and American regulatory bodies, industry representatives and academic clinical research organizations met in Dublin and Washington to define new criteria for early and late stent thrombosis, as well as cardiac and non-cardiac death, myocardial infarction and revascularization. A uniform methodological approach such as this might elucidate the true incidence of the late stent thrombosis phenomenon. In the meantime, a clinical investigation into the duration of dual antiplatelet therapy should be seriously considered; however, such a study would necessitate a huge cohort of patients. 